\title{
Prediction and investigation between impeller blade shape parameters and performance parameters in CFD and ANN
}

\author{
Faye Jin ${ }^{a, b}, \operatorname{Ran}_{T^{2} o^{a, b^{*}}}$ and Ruofu Xiao ${ }^{a, b}$
}

${ }^{a}$ College of Water Resources and Civil Engineering, China Agricultural University, 17 Qinghua Donglu, Beijing 100083, People’s Republic of China ${ }^{b}$ Beijing Engineering Research Center of Safety and Energy Saving Technology for Water Supply Network System, Beijing 100083, China

\begin{tabular}{|c|c|}
\hline $\mathbf{A} \mathbf{R}$ & A B S T RAC T \\
\hline Article history: & \multirow{10}{*}{$\begin{array}{l}\text { The blade shape parameters have a remarkable effect on the centrifugal pump performance. In order } \\
\text { to reveal the relationship between these parameters and pump performance, a single channel was } \\
\text { regarded as the research object to calculate its performance by numerical simulation, and the } \\
\text { performance was measured on an experimental rig. The optimized ANN is proposed, and it is proved } \\
\text { to be highly accurate. The ANN correlation coefficient of the total response could be above } 0.997 \text { after } \\
\text { thousands of retaining. The sorts and degrees affecting performance parameters were found out by } \\
\text { gray relation analysis. It was found that the blade angles at the leading edge were more influential for } \\
\text { reaction force, head and minimum pressure, while the wrap angles had greater impact for efficiency. } \\
\text { Furthermore, a multiple linear regression model was established to quantify the weight and trend of } \\
\text { the influence of blade shape parameters on performance. The results provide a reference guide for the } \\
\text { optimized design of centrifugal impeller to improve pump performance. }\end{array}$} \\
\hline & \\
\hline 2021 & \\
\hline & \\
\hline$\frac{11 \mathrm{D}}{K e v w}$ & \\
\hline & \\
\hline Blade angle & \\
\hline & \\
\hline Reac & \\
\hline & \\
\hline
\end{tabular}

(C) 2022 Growing Science Ltd. All rights reserved.

\section{Introduction}

Impeller is the key component to transfer the mechanical energy of prime mover to the transported liquid, and blades play a major role in hydraulic performance in centrifugal pumps. An appropriate blade design can not only improve excellent hydraulic performance, but also help to prolong long life. In practical engineering application, changing of the blade shape parameters to meet the design requirements is a simple, easy, rapid and effective way. Hence, the effect of each or several blade angles is of great importance to optimize the overall performance of the impeller. The axial force mainly includes the cover plate force generated by the clearance fluid surrounding the impeller and the dynamic reaction force generated by the fluid in the inner channel of the impeller. The dynamic reaction force is difficult to be measured by experiment. So, computational fluid dynamics (CFD) is an important method to study the dynamic reaction force. Shi et al. (2009) predicted the axial force of submersible pumps based on CFD and analyzed the composition of axial force. The ratio of the axial force on the inner surface of the impeller to the total axial force is about 0.8 . Hou et al. (2017) conducted an experimental study on the axial force of the rotor of the twin-screw compressor. They provided a new idea for the experiment of measuring axial force. The axial force is calculated by mean pressure model and sector pressure model, and compared with the experimental results. In some special cases, such as blade cavitation, it will also have a certain impact on the axial force of the impeller. Pump performance parameters, such as head and efficiency, are the most basic parameters to evaluate pump performance. As we all know, the blade trailing-edge angle increases, and the head will be increased according to the Euler formula. The theoretical head formula of the pump was also mainly deduced from the Euler equation. On the other hand, it would have an impact on efficiency. De Souza et al. (2008) studied the influence of main geometric parameters of single blade on * Corresponding author.

E-mail addresses: randytao@,cau.edu.cn (R. Tao) 
performance based on response surface methodology (RSM) and CFD method. The research parameters are impeller width, blade leading-edge angle, blade wrap angle and blade inlet thickness. The research results showed that the flow area could increase by increasing impeller width, reducing blade leading-edge angle, reducing the wrap angle and inlet thickness. Nishi et al. (2012) experimentally studied the influence of the impeller outlet width on the performance. At the partial flow rate, the head and efficiency decrease with the increase of outlet width, but on the contrary at the high flow rate.

In addition, the minimum pressure of the impeller also plays an important role in judging the cavitation performance. The range of attack angle with good cavitation performance is generally taken as $\Delta \beta=3^{\circ} \sim 10^{\circ}$. Within this range, the attack angle has little impact on the efficiency of the pump, but it can improve the cavitation performance of the pump (Guan 2011; Badr and Ahmed 2015). In order to further clarify the relationship between blade shape and impeller performance parameters, this study was carried out. With the improvement of computers and the development of artificial neural networks (ANN), the results of ANN calculation have been comparable to those predicted by CFD. Many scholars have applied ANN to accurately predict the performance of centrifugal pumps. Wang et al. (2019) proposed an optimization method to reduce axial force. The hydraulic efficiency and the sufficient pump head were improved. Zhu et al. (2020) established an artificial neural network through the global dynamic criterion algorithm and proposed an effective method to reduce the axial force. This method improves the adverse effect of axial force on the pump by setting balance holes and balance plates. Also, it has no obvious effect on the pump performance. Grey relation analysis (GRA) is a method of data correlation degree proposed by Ju-Long (1982a). It plays an important role in statistics. This analysis method is helpful to the influence relationship between each blade shape parameter and pump performance since there is a complex nonlinear relationship between the performance of a centrifugal pump and the impeller blade parameters. According to the GRA analyses of this research, it is found that the blade angles at leading edge were more influential for reaction force, head and minimum pressure, while the wrap angles have greater impact for efficiency.

\section{Theory and Method}

\subsection{Turbulence Model and Governing Equations}

The flow in the centrifugal pump is a kind of incompressible three-dimensional turbulent flow. The variables of pressure and force could be calculated accurately by the SST $k-\omega$ model (Larsen \& Fuhrman 2018), which combines $k-\varepsilon$ in free flow and $k-\omega$ near the wall (Menter 1993). The turbulence model can simulate strong cyclones and improve the sensitivity to the inverse pressure gradient. The transport equations of the turbulent kinetic energy $k$ and specific dissipation rate $\omega$ are expressed as:

$$
\begin{aligned}
& \frac{\partial(\rho k)}{\partial t}+\frac{\partial\left(\rho u_{i} k\right)}{\partial x_{i}}=P-\frac{\rho k^{3 / 2}}{l_{k-\omega}}+\frac{\partial}{\partial x_{i}}\left[\left(\mu+\sigma_{k} \mu_{t}\right) \frac{\partial k}{\partial x_{i}}\right] \\
& \frac{\partial(\rho \omega)}{\partial t}+\frac{\partial\left(\rho u_{i} \omega\right)}{\partial x_{i}}=C_{\omega} P-\beta \rho \omega^{2}+\frac{\partial}{\partial x_{i}}\left[\left(\mu_{l}+\sigma_{\omega} \mu_{t}\right) \frac{\partial \omega}{\partial x_{i}}\right]+2\left(1-F_{1}\right) \frac{\rho \sigma_{\omega 2}}{\omega} \frac{\partial k}{\partial x_{i}} \frac{\partial \omega}{\partial x_{i}}
\end{aligned}
$$

where, $\rho$ is the fluid density, $P$ is the turbulence generation term, $\mu$ is the dynamic viscosity, $\mu_{t}$ is the eddy viscosity coefficient, $\sigma$ is the model constant, $C_{\omega}$ is the coefficient of turbulent dissipation term, $F_{1}$ is the blending equation, $l_{k-\omega}$ is the scale of turbulence. Hence, it is a low Reynolds number model, which is similar to $k-\omega$ model in the requirements of grid (Menter, 2006; Launder and Sharma 1974; Wilcox 1988). The calculation method near the wall is the wall function. It is assumed that the logarithmic profile reasonably approximates the velocity distribution near the wall and it provides a means to numerically compute the fluid shear stress from the wall to the given distance.

\subsection{The Performance Parameters of the Impeller}

\subsubsection{The reaction force of impeller}

The liquid usually flows into the impeller in the axial direction and flows out in the radial or oblique direction. The direction of liquid flow through the impeller changes because the liquid is subjected to the force of the impeller. On the contrary, the liquid gives the impeller a reaction force with equal size and opposite direction. This force points the direction of inlet flow and is determined by the momentum theorem:

$$
F_{d}=\rho Q_{t}\left(v_{\text {in }}-v_{\text {out }} \cos \alpha\right)
$$

where $Q_{t}$ is the flow rate, $\mathrm{m}^{3} / \mathrm{s} ; \rho$ is the density of the fluid, $\mathrm{kg} / \mathrm{m}^{3} ; v_{\text {in }}$ and $v_{\text {out }}$ are the velocity of inlet and outlet of impeller, $\mathrm{m} / \mathrm{s} . \alpha$ is the angle between the impeller outlet shaft velocity and the axial direction shown as the Fig. 1. 


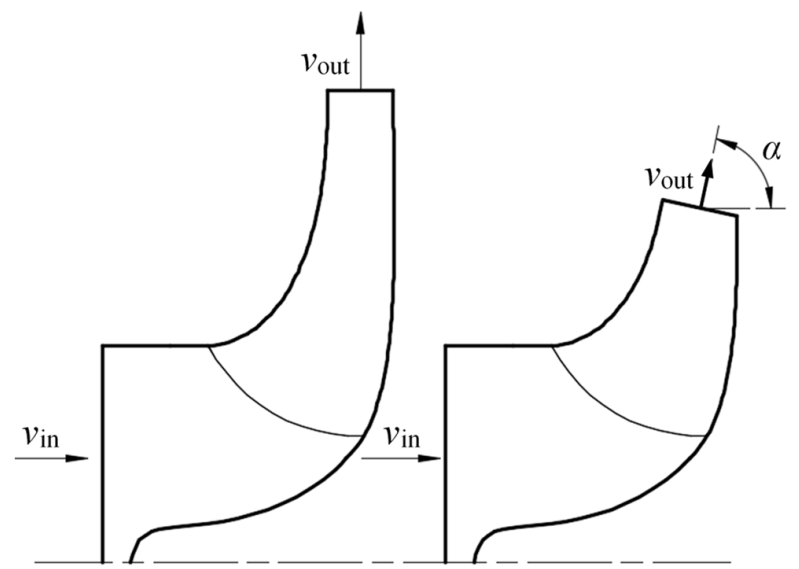

Fig. 1. The reaction force acting on the impeller

\subsubsection{Head}

For the theoretical head, the viscous force is not considered, so there is only the acting moment of the blade on the liquid. The impeller transmits the torque to the liquid through the blade to increase the energy. In this study, the inlet flow considered is a swirling flow. Thus, Euler equation of the impeller is expressed as:

$$
H_{t}=\frac{1}{g}\left(u_{2} v_{u 2}-u_{1} v_{u 1}\right) \text {, }
$$

where $v_{u 1}=u_{1}-v_{\mathrm{m} 1} \cot \beta_{1}, v_{u 2}=u_{2}-v_{\mathrm{m} 2} \cot \beta_{2}, u_{1}=r_{1} \pi n / 30, u_{2}=r_{2} \pi n / 30, \beta_{1}, \beta_{2}$ is the leading-edge blade angle and the trailing edge blade angle, respectively. Pump efficiency is the ratio of the actual power of the pump to the power provided by the motor feed pump:

$$
\eta=\frac{\rho g Q H}{P_{s}} \times 100 \%
$$

where, $H$ is the effective head of the pump, that is, the energy obtained from the pump by a unit amount of liquid in the gravity field, $\mathrm{m} ; Q$ is actual flow rate of pump, $\mathrm{m}^{3} / \mathrm{s} ; P_{s}$ is the power of the motor, W.

\subsection{Data Analysis Methods}

GRA is a multi-factor statistical analysis method. It establishes the parent sequence that changes with time, and takes the change of each evaluation object with time as a sub sequence. The conclusion would be calculated according to the correlation between each sub sequence and the parent sequence. The concept of the gray system is put forward relative to white system and black system. It would judge the importance of factors affecting the development of the system. Another function is required to comprehensively evaluate problems and give the ranking of research objects or schemes. The calculation formula of gray correlation coefficient is: on the liquid. The impeller transmits the torque to the liquid through the blade to increase the energy. In this study, the inlet flow considered is a swirling flow. Thus, Euler equation of the impeller is expressed as:

$$
\xi_{i}(k)=\frac{\min _{i} \min _{k}\left|y(k)-x_{i}(k)\right|+\rho \max _{i} \max _{k}\left|y(k)-x_{i}(k)\right|}{\left|y(k)-x_{i}(k)\right|+\rho \max _{i} \max _{k}\left|y(k)-x_{i}(k)\right|}
$$

where, $\min _{i} \min _{k}\left|y(k)-x_{i}(k)\right|$ is the absolute value of the second-order minimum difference between $y$ and $x_{i}$, $\max _{i} \max _{k}\left|y(k)-x_{i}(k)\right|$ is the absolute value of the second-order maximum difference between $y$ and $x_{i}$ at point $k . \rho$ is grey resolution coefficient, whose value is between $0 \sim 1$. The correlation degree would be obtained by substituting the correlation coefficient into the above formula (Ju-Long 1982b):

$$
r_{i}=\frac{1}{n} \sum_{k=1}^{n} \xi_{i}(k) \text {. }
$$

Latin hypercube sampling (LHS) was applied in the study of (Olsson et al. 2003; Sen-Chun et al. 2020). It is a kind of stratified random sampling, which can efficiently sample from the distribution interval of variables. $N$ samples need to be taken from the specified interval. Each variable is divided into the same $N$ smaller interval. A value was randomly selected from each interval. This method is different from random sampling. It could ensure the full coverage of each variable range. 


\subsection{Strategy}

In this study, the artificial neural network (ANN) was used as the basic research method, and a centrifugal pump was used as the research object to study the influence of different blade angle and wrap angle on the performance of centrifugal pump. The process sketch map of this research was shown in Fig. 2. Firstly, the impeller blade was parameterized to 9 parameters,

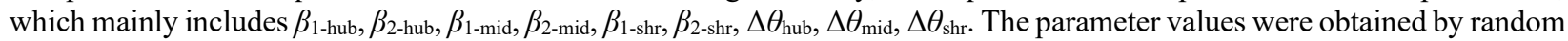
assignment with LHS. Thus, 100 random blade samples were simulated to get pump impeller performance by computational fluid dynamics (CFD). Then the simulated results were compared with the experimental results to validate the calculating accuracy. Secondly, the simulated results were trained in optimized ANN to get more samples and results. The response correlation coefficient $R$ was regarded as judging criteria in the ANN. If $R$ is not larger than $R_{s}$, the network would be regenerated until $R>R_{S} .1000$ random samples be predicted in the optimized ANN in the study. In addition, several ANN results were compared with CFD results to test the ANN accuracy. Finally, the relationship between input parameters and output parameters head $H$, reaction force $F_{d}$ and impeller efficiency $\eta$ was summarized by grey relation analysis (GRA).

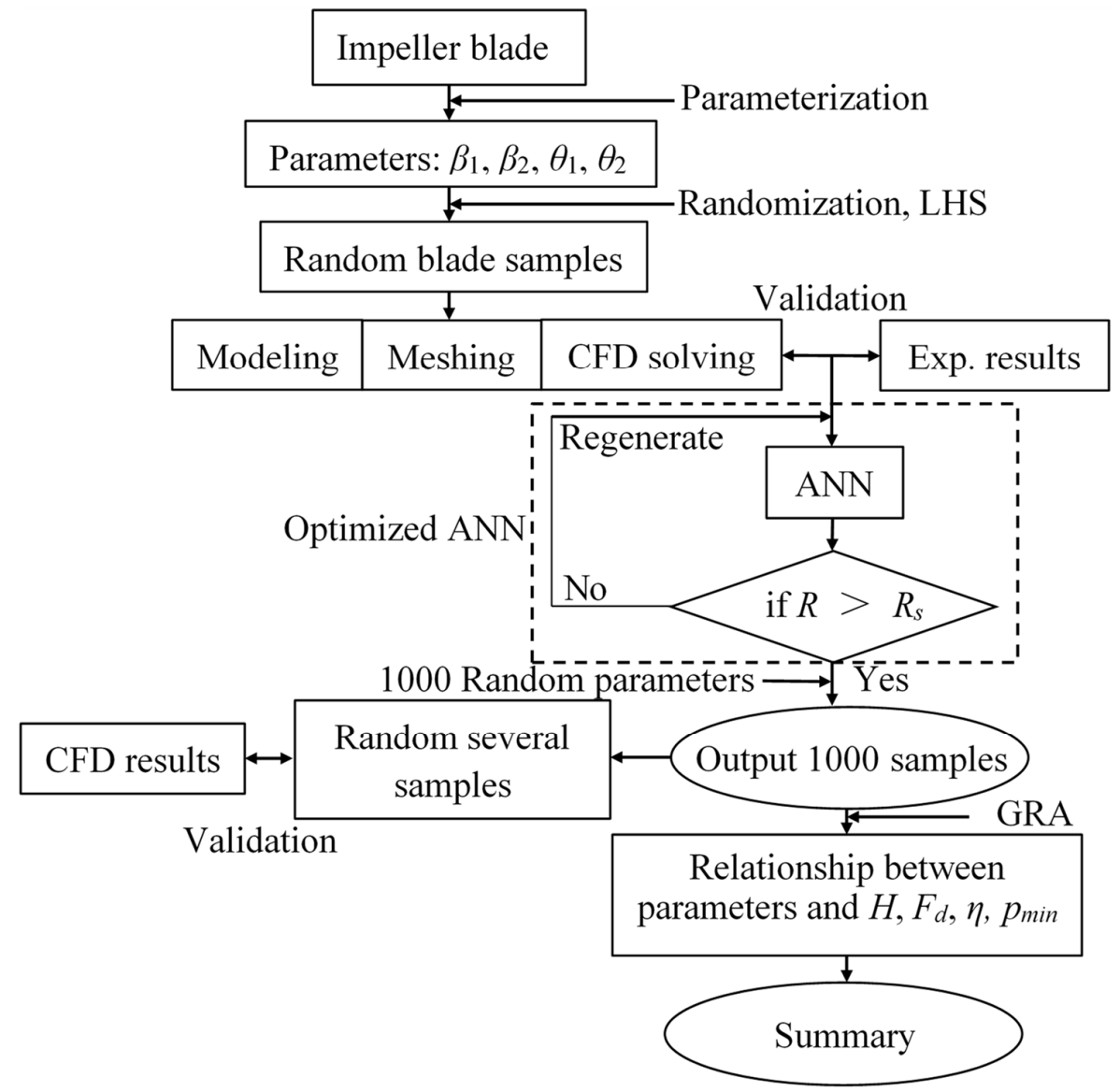

Fig. 2. The process sketch map of the strategy

\section{Pump Model and Experimental Setup}

\subsection{Pump Model Description}

In order to obtain a lot of blade samples and reduce calculating time cost, single blade channels are used to evaluate performance in a simplified model. The initial pump impeller consists of 6 blades. The special speed of this pump is defined as following:

$$
n_{s}=\frac{3.65 n_{d} \sqrt{Q_{d}}}{H_{d}^{3 / 4}},
$$

where, $n_{d}$ is design rotational speed; $Q_{d}$ is design flow rate; $H_{d}$ is design head. The impeller structure model in the study is shown as Fig. 3. The main parameter values of the originally designed impeller are listed in detail in Table 1. 


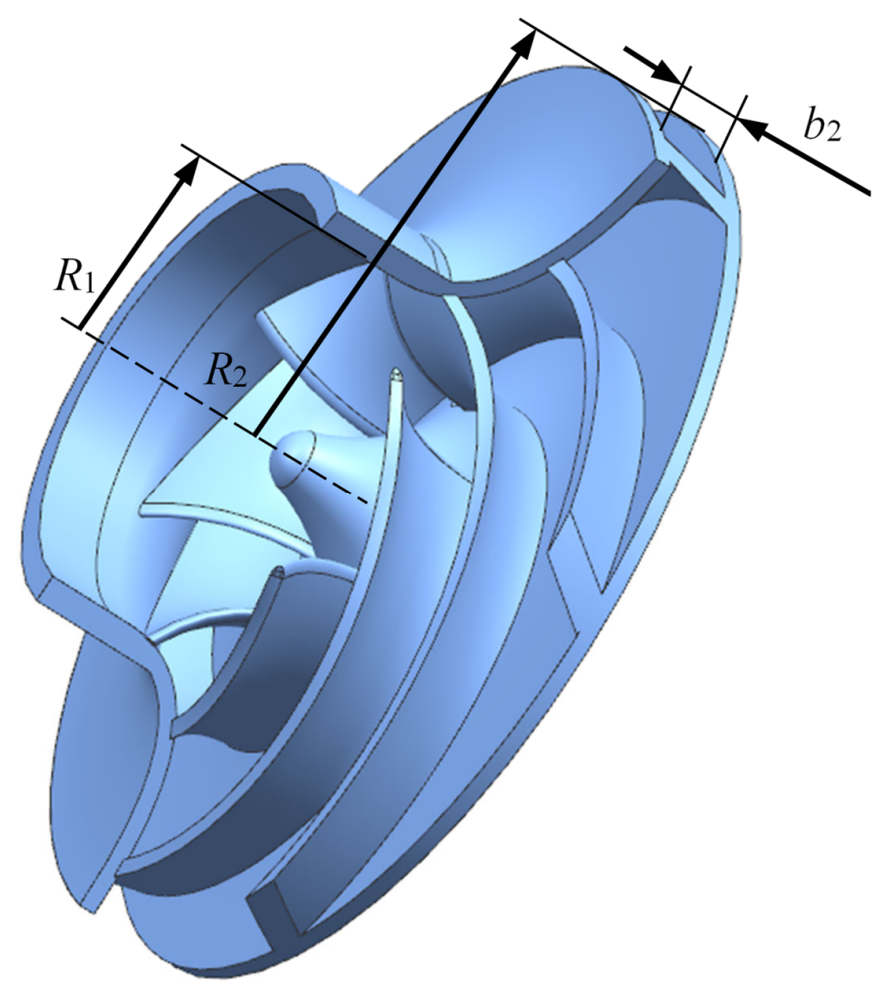

Fig. 3. The initial impeller structure model

Table 1. Main parameters and values of the pump

\begin{tabular}{lll}
\hline Parameters & Symbols & Value \\
\hline Design Flow Rate & $Q_{d}$ & $200\left[\mathrm{~m}^{3} / \mathrm{h}\right]$ \\
Design Head & $H_{d}$ & $92[\mathrm{~m}]$ \\
Design Rotational Speed & $n_{d}$ & $2950[\mathrm{r} / \mathrm{min}]$ \\
Impeller Blade Number & $z$ & $6[-]$ \\
Impeller Outlet Radius & $R_{2}$ & $136.0[\mathrm{~mm}]$ \\
Impeller Inlet Radius & $R_{1}$ & $62.5[\mathrm{~mm}]$ \\
Impeller Outlet Width & $b_{2}$ & $19.0[\mathrm{~mm}]$ \\
Specific speed & $n_{s}$ & $85.4[-]$ \\
\hline
\end{tabular}

In order to study the effects of leading-edge blade angle and trailing-edge blade angle on the streamlines to pump performance, the blade three-dimensional model is parameterized graphically. In the meridional passage, there are three streamlines divided, which are named shr, mid and hub, respectively, as shown in the Fig. 4(a). On each streamline, the main blade parameters include the leading-edge blade angle $\beta_{1}$, the trailing edge blade angle $\beta_{2}$, leading edge wrap angle $\theta_{1}$, and trailing edge blade angle $\theta_{2}$, as shown in the Fig. 4(b). The subscripts with "shr", "mid" and "hub" indicate their position. Generally, the wrap angle is usually expressed in the form of difference:

$$
\Delta \theta=\theta_{2}-\theta_{1}
$$

The details of parameters of the original impeller are listed in Table 2.

Table 2. Impeller blade angles and wrap angles

\begin{tabular}{cccc}
\hline Parameters & Hub & Mid-span & Shroud \\
\hline$\beta_{1}\left[^{\circ}\right]$ & 25.3 & 25.4 & 19.2 \\
$\beta_{2}\left[^{\circ}\right]$ & 21.9 & 21.9 & 21.8 \\
$\theta_{1}\left[{ }^{\circ}\right]$ & 0 & 0 & 0 \\
$\theta_{2}\left[{ }^{\circ}\right]$ & 152.0 & 149.0 & 146.0 \\
\hline
\end{tabular}




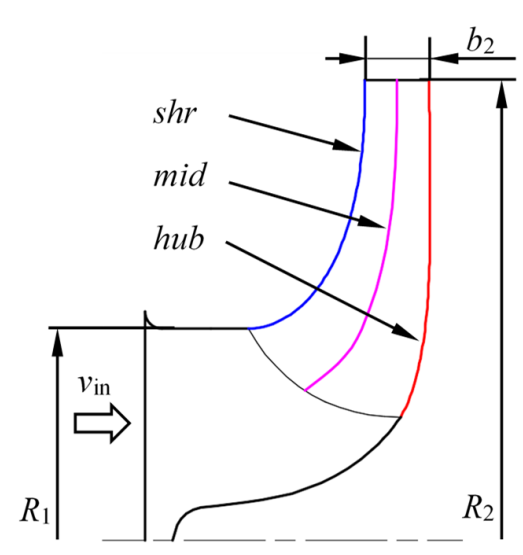

(a) Meridional view

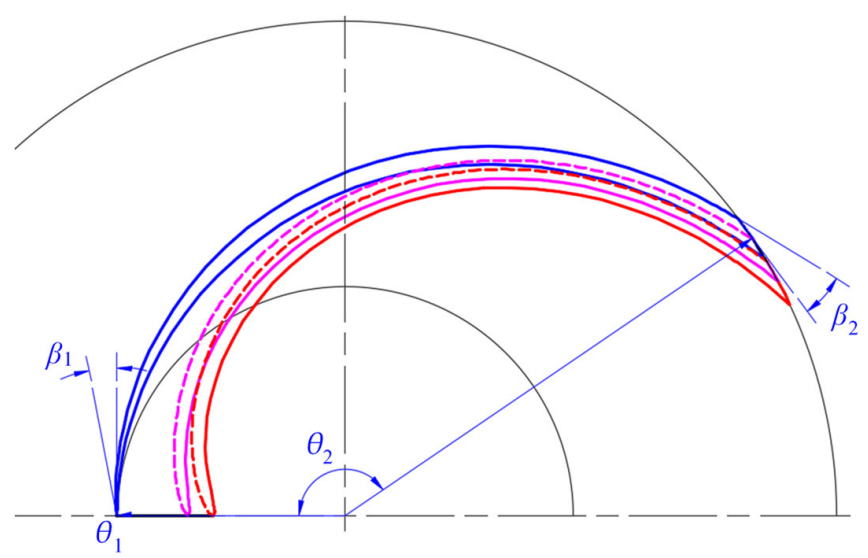

(b) Axial view

Fig. 4. The meridional and axial view of blade parameterization

\subsection{Computational Mesh Check and CFD Setup}

Refinement factors $r_{21}$ and $r_{32}$ are larger than 1.3. The parameters that head $H, F_{\text {blade }}, F_{h u b}$ and $F_{\text {shr }}$ of a single flow channel are applied as evaluation criteria which are listed in Table 2. The local order of accuracy $p$ calculated ranges from 3.04 to 9.40. The approximate relative error $e_{\mathrm{a}}^{21}$ is in the range from $0.005 \%$ to $0.335 \%$, and $e_{\mathrm{a}}^{32}$ is in the range from $0.076 \%$ to $0.148 \%$. The discretization uncertainty $e_{\mathrm{ext}}^{21}$ is in the range from $0.001 \%$ to $0.084 \%$, and $e_{\mathrm{ext}}^{32}$ is in the range from $0.006 \%$ to $0.102 \%$. The convergence index of $\mathrm{M}_{1} \mathrm{GCI}_{\text {fine }}^{21}$ is in the range from $0.001 \%$ to $0.105 \%$. It is necessary to note that less grid is conducive to saving computing resources while more grids can ensure the calculation accuracy. Hence, M2 is selected as a compromise between accuracy and computational cost with 264,244 nodes for a single channel.

The computation mesh of the centrifugal pump model is shown in Fig. 5. The boundaries consist of mass flow inlet, static pressure outlet and rotational periodicity between flow channels. For the finite volume spatial discretization, high quality hexahedral grids are adopted to discretize the channel domains. Boundary layer meshing of the blades is considered, and leading-edge and trailing-edge surfaces are also locally refined, as shown in Fig. 5. Grid convergence index (GCI) based on Richardson extrapolation method is used to analyze the grid convergence Celik et al. (2008). Three meshing schemes, M1, M2, and M3, are determined proportionally, with nodes of 109,250, 264,244 and 584,272, respectively.

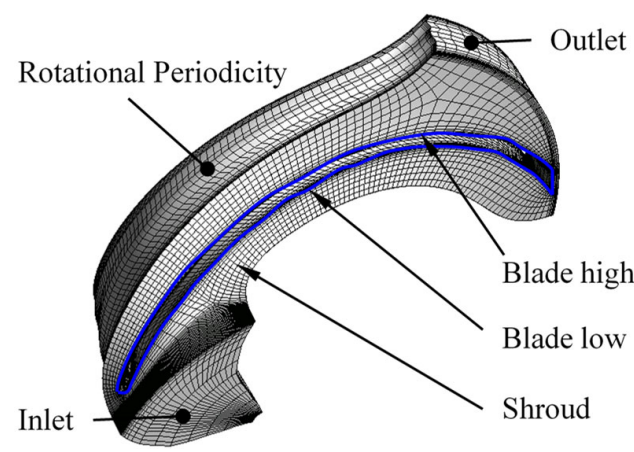

(a) Domain mesh view I

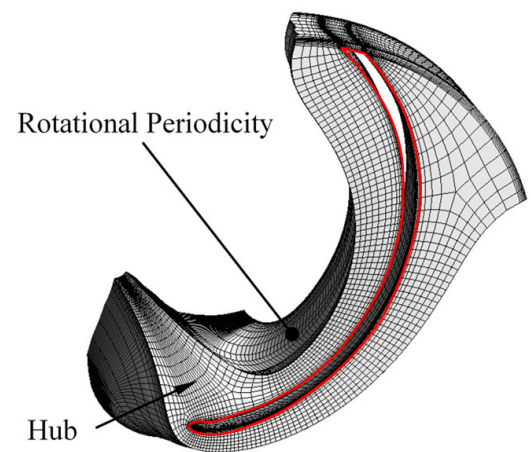

(b) Domain mesh view II

Fig. 5. Domain mesh of impeller passage

CFD simulation was based on the commercial code of ANSYS CFX. Single blade channel was rotating reference frames, the speed of which was set as $2950 \mathrm{r} / \mathrm{min}$. In order to analyze the influence of different blade parameters on impeller performance, the designed flow rate $Q$ was calculated respectively. The reference pressure was set to 101,325 Pa. No-slip condition is employed for all wall surfaces. The convergence residual standard is set to $1.0 \times 10^{-5}$ in this case. The local parallel computing is used for completing the algorithm process. The central difference scheme is used for the diffusion term for the discretization schemes. The second-order upwind scheme is applied for the convective term. 
Table 2. Evaluation results of discretization error in GCI check

\begin{tabular}{ccccc}
\hline & $H[\mathrm{~m}]$ & $F_{\text {blade }}[\mathrm{N}]$ & $F_{\text {hub }}[\mathrm{N}]$ & $F_{\text {shr }}[\mathrm{N}]$ \\
\hline$\phi_{1}$ & 95.55 & -371.56 & 3322.55 & -2932.42 \\
$\phi_{2}$ & 95.23 & -371.77 & 3322.73 & -2933.10 \\
$\phi_{3}$ & 95.15 & -372.32 & 3325.66 & -2935.34 \\
$p$ & 6.08 & 3.04 & 9.40 & 3.85 \\
$\phi_{\text {ext }}^{21}$ & 95.63 & -371.39 & 3322.53 & -2932.03 \\
$e_{a}^{21}$ & $0.335 \%$ & $0.057 \%$ & $0.005 \%$ & $0.023 \%$ \\
$e_{\text {ext }}^{21}$ & $0.084 \%$ & $0.046 \%$ & $0.001 \%$ & $0.013 \%$ \\
$\mathrm{GCl}_{\text {fine }}^{21}$ & $0.105 \%$ & $0.057 \%$ & $0.001 \%$ & $0.016 \%$ \\
$\phi_{\text {ext }}^{32}$ & 95.25 & -371.39 & 3322.53 & -2932.03 \\
$e_{a}^{32}$ & $0.084 \%$ & $0.148 \%$ & $0.088 \%$ & $0.076 \%$ \\
$e_{\text {ext }}^{32}$ & $0.017 \%$ & $0.102 \%$ & $0.006 \%$ & $0.036 \%$ \\
$\mathrm{GCl}_{\text {fine }}^{32}$ & $0.021 \%$ & $0.128 \%$ & $0.007 \%$ & $0.045 \%$ \\
\hline
\end{tabular}

\subsection{Experimental Setup}

The experimental system for testing the performance of the centrifugal pump was shown in Fig. 6. There were two pressure sensors fixed at the inlet and outlet of the tested pump to monitor the head $H$. A valve was set at the outlet of the centrifugal pump. One was installed on the inlet of the fluid tank to control the flow of the pipeline system, and another valve was arranged in the pipeline to be convenient for disassembly and assembly. A flow meter and a circulating pump were set to monitor the flow rate of the pipeline system. Pressure, flow and other signals were transmitted to the terminal to collect and record.

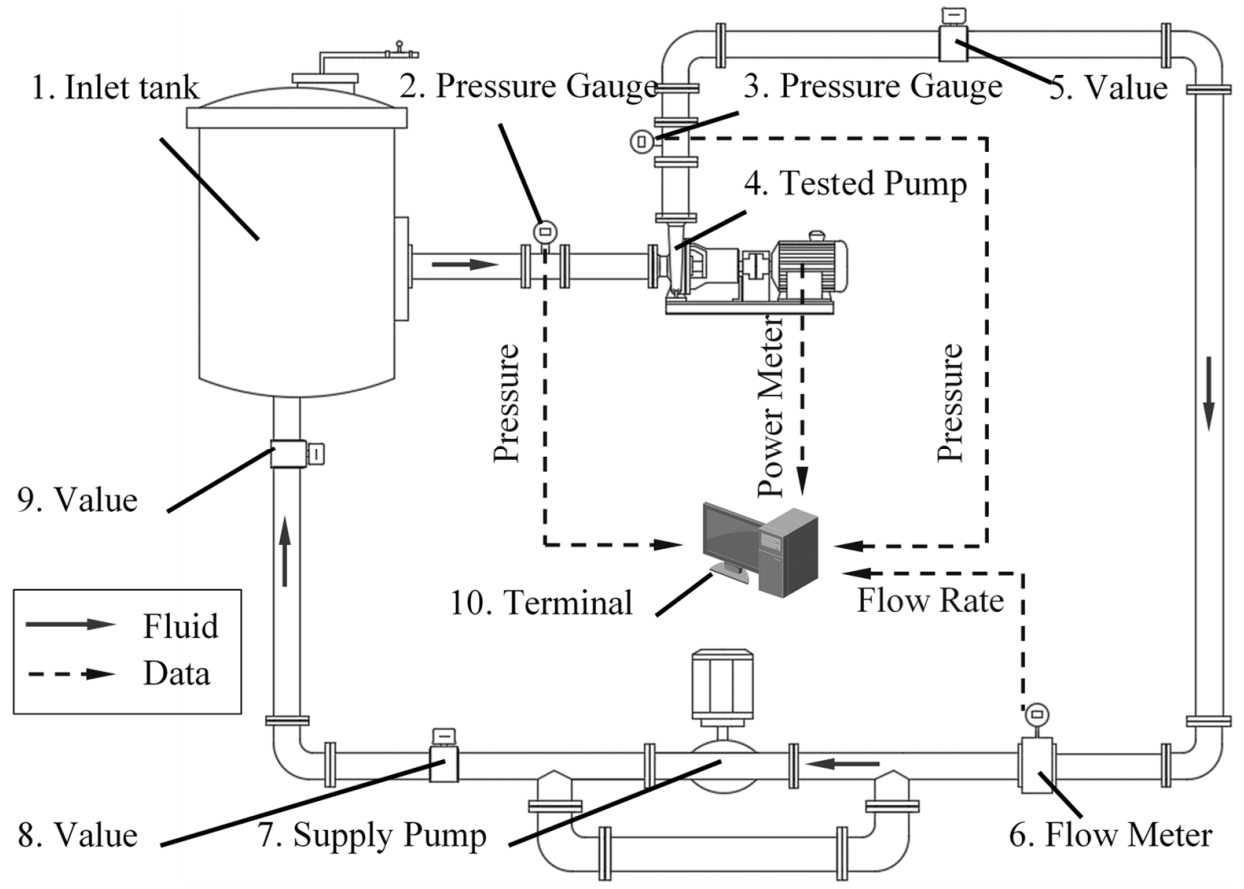

Fig. 6. Test rig of experimental system.

\subsection{Validation of Numerical Simulation Results}

The numerical results of the pump performance are compared with the experimental results in Fig. 7 to verify the simulation accuracy. It was found that the head calculated was higher than the experimental head. At $Q=120 \mathrm{~m}^{3} / \mathrm{h}, 160 \mathrm{~m}^{3} / \mathrm{h}$, $200 \mathrm{~m}^{3} / \mathrm{h}$ and $240 \mathrm{~m}^{3} / \mathrm{h}$, the predicted $H$ has small deviations of $2.45 \%, 3.41 \%, 6.34 \%$ and $7.83 \%$ from the experimental data. 
The predicted $P$ values are slightly smaller than the experimental results, but the trends are the same. At the above flow rate, the errors between the predicted $P$ and the experimental values were $3.76 \%, 5.03 \%, 4.61 \%$ and $5.04 \%$, respectively.

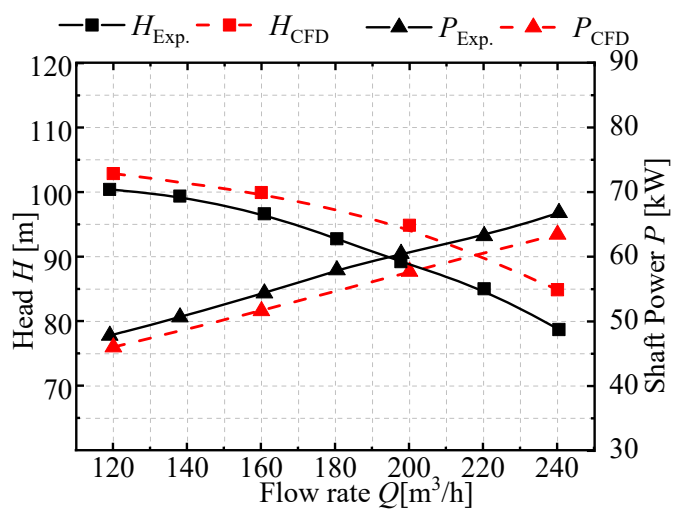

Fig. 7. Performance curves comparison

\section{Results and Analysis}

\subsection{ANN Setup and Validation}

It is more important and meaningful to ensure CFD and machine learning methods for effective simulation and accurate prediction for a lot of samples. It is a time-consuming and arduous work to calculate the performance of the pump by CFD commercial software. The impeller calculating domain has been simplified as soon as possible. But it still costs a lot of time to obtain a CFD solution. Artificial neural network (ANN) is a widely used machine learning system, which is inspired by the biological nervous system. The joint solution method of ANN and CFD was proved to be efficient. A neural network of output parameters and input parameters was established based on CFD initial samples. In this study, Bayesian regulation was chosen as the training algorithm, which requires more time, but can result in good generalization for difficult, small or noise datasets. The artificial neural network has 10 hidden layers. The input layer is 6 parameters of blade angle and 3 parameters of wrap angle on the hub, mid span and shroud streamline, respectively. The value ranges of input parameters were listed in Table 3 . The output layer has four parameters which includes head $H$, efficiency $\eta$ and reaction force $F_{z} .70 \%$ samples are selected to train, $15 \%$ samples are selected to validate, and $15 \%$ samples are selected to test.

Different solutions may be generated due to different initial weights and bias values when the ANN is trained. In addition, the data is divided into training sets, verification sets and test sets in different ways, which may also produce different solutions. Therefore, different ANN trained for the same problem may give different outputs even if the same data were input. In order to find a neural network with good accuracy, it needs to be retrained many times. Linear regression is carried out between the network output and the target. The number of neural network retraining exceeds 1000, and the correlation coefficient $R$ of the total response was above 0.997 .

The prediction result of optimized ANN becomes almost as accurate as that of computational fluid dynamics, and the errors between them were less than $5 \%$ for $F_{z}$, less than $1 \%$ for $\eta$ and about $2 \%$ for $H$. The validating sample error was about $14 \%$ for $p_{\text {min }}$, while the other error is less than $10 \%$, as shown in the Fig. 8 . The optimized ANN was then applied as an alternative to CFD. The time cost is greatly reduced, and it takes only 2-3 seconds to calculate the results of 1000 samples by optimized ANN.

Table 3. The value ranges of input parameters

\begin{tabular}{|c|c|c|c|}
\hline Parameters & $\mathrm{Hub}$ & Mid-span & Shroud \\
\hline$\beta_{1}\left[{ }^{\circ}\right]$ & $\begin{array}{r}25.3(+5) \\
(-5)\end{array}$ & $\begin{array}{r}25.4(+5) \\
(-5)\end{array}$ & $\begin{array}{r}19.2(+5) \\
(-5)\end{array}$ \\
\hline$\beta_{2}\left[{ }^{\circ}\right]$ & $\begin{array}{r}21.9(+5) \\
(-5)\end{array}$ & $\begin{array}{r}21.9(+5) \\
(-5)\end{array}$ & $\begin{array}{r}21.8(+5) \\
(-5)\end{array}$ \\
\hline$\theta_{1}\left[{ }^{\circ}\right]$ & $\begin{array}{c}0(+5) \\
(-5)\end{array}$ & $\begin{array}{c}0(+5) \\
(-5)\end{array}$ & $\begin{array}{c}0(+5) \\
(-5)\end{array}$ \\
\hline$\theta_{2}\left[^{\circ}\right]$ & $\begin{array}{r}152.0(0) \\
(-5)\end{array}$ & $\begin{array}{r}149.0(+5) \\
(-5)\end{array}$ & $\begin{array}{c}146.0(+5) \\
(0)\end{array}$ \\
\hline
\end{tabular}




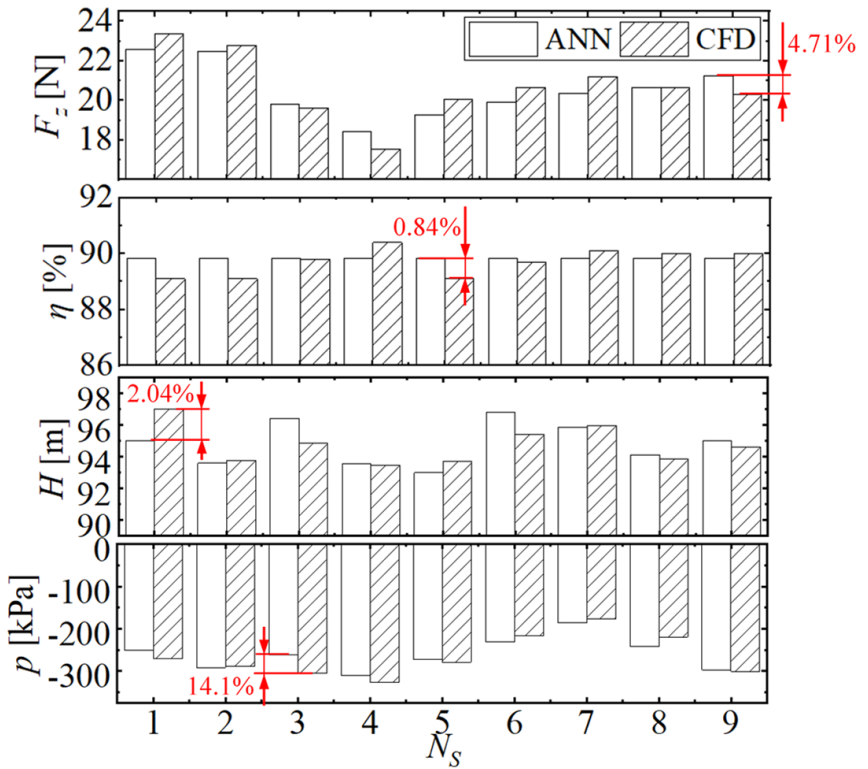

Fig. 8. The comparison of optimized ANN and CFD

\subsection{Influence of Blade Wrap Angle and Installation Angle}

The influencing degree of input 9 parameters on the impeller performance was listed in Table 4 . The value column represented gray relation degree, and the sort stood for the relation degree sort. For the clearer explanation, it is divided into the following levels according to the sort of gray relation degree. The parameters ranked 1, 2, 3 are called significant correlation. The parameters ranked 4, 5, 6 are called sub-significant correlation and the parameters ranked 7, 8, 9 are called weak significant correlation.

As for $F_{z}$, the gray relation degree is almost the same, while the significant correlation parameters are $\beta_{1-h u b}, \beta_{1-s h r}$ and $\beta_{2-}$ shr. The weak significant correlation parameters are $\beta_{1-\text { hub }}, \beta_{1 \text {-shr }}$ and $\beta_{2 \text {-shr. }} \Delta \theta_{\text {mid }}, \Delta \theta_{\text {shr }}$ and $\beta_{2 \text {-mid. }}$. It indicates blade angle is more influential than the wrap angle in general. As for $H$, the significant correlation parameters are $\beta_{1 \text {-shr }}, \beta_{1-h u b}$ and $\beta_{1 \text {-mid. }}$. The sub-significant correlation parameters are $\Delta \theta_{\text {mid }}, \Delta \theta_{\text {shr }}$, and $\Delta \theta_{\text {hub }}$. The head will raise with increasing blade angle at the trailing edge, which is only for inlet flow without rotation. In fact, the blade angle at the leading edge is more influential than the blade angle at the trailing edge. As for $\eta$, the gray relation degree differences among the parameters are relatively large. The significant correlation parameters are $\Delta \theta_{\text {mid }}, \Delta \theta_{\text {shr }}$, and $\Delta \theta_{h u b}$. It demonstrates that the wrap angle could be adjusted appropriately to improve the efficiency. As for $p_{\min }$, the significant correlation parameters are $\beta_{1-\text { mid }}, \beta_{1-s h r}$ and $\Delta \theta_{h u b}$. The main reason is that the lowest pressure point is located at the inlet of the impeller. Cavitation is more likely to occur at the junction of the hub and blade due to the impact of flow lash.

Table 4. Grey relation degree of the output parameters and input parameters

\begin{tabular}{c|cc|cc|cccc}
\hline \multirow{2}{*}{ Parameter } & \multicolumn{2}{|c|}{$F_{z}$} & \multicolumn{2}{c|}{$H$} & \multicolumn{2}{c}{$\eta$} & \multicolumn{2}{c}{$p_{\min }$} \\
& Value & Sort & Value & Sort & Value & Sort & Value \\
\hline$\beta_{1-h u b}$ & 0.871174 & 1 & 0.966486 & 2 & 0.6482 & 5 & 0.744970 \\
$\beta_{2-h u b}$ & 0.871140 & 5 & 0.966477 & 8 & 0.632266 & 6 & 0.744969 & 6 \\
$\beta_{1-\text { mid }}$ & 0.871147 & 4 & 0.966485 & 3 & 0.651889 & 4 & 0.744994 & 1 \\
$\beta_{2-\text { mid }}$ & 0.870999 & 9 & 0.966467 & 9 & 0.630256 & 8 & 0.744959 & 9 \\
$\beta_{1-\text { shr }}$ & 0.871153 & 2 & 0.966487 & 1 & 0.619137 & 9 & 0.744987 & 2 \\
$\beta_{2-\text { shr }}$ & 0.871151 & 3 & 0.966480 & 7 & 0.630559 & 7 & 0.744968 & 8 \\
$\Delta \theta_{\text {hub }}$ & 0.871137 & 6 & 0.966482 & 6 & 0.653196 & 3 & 0.744970 & 3 \\
$\Delta \theta_{\text {mid }}$ & 0.871115 & 7 & 0.966484 & 4 & 0.656676 & 1 & 0.744970 & 4 \\
$\Delta \theta_{\text {shr }}$ & 0.871108 & 8 & 0.966483 & 5 & 0.654163 & 2 & 0.744968 & 7 \\
\hline
\end{tabular}

According to the above analysis, it was found that the impeller performance is influenced largely by the blade shape. In order to explore the corresponding relationship more clearly between performance parameters and blade shape, a multiple linear regression model was established between them. The model relationship of the performance parameters and each blade parameter can be expressed as following:

$$
\begin{aligned}
& F_{z}=a_{1} \beta_{1-h u b}+a_{2} \beta_{2-h u b}+a_{3} \beta_{1-m i d}+a_{4} \beta_{2-m i d}+a_{5} \beta_{1-s h r}+a_{6} \beta_{2-s h r} \\
& +a_{7} \Delta \theta_{h u b}+a_{8} \Delta \theta_{\text {mid }}+a_{9} \Delta \theta_{\text {shr }}+a_{10}
\end{aligned}
$$




$$
\begin{aligned}
& H=b_{1} \cot \beta_{1-h u b}+b_{2} \cot \beta_{2-h u b}+b_{3} \cot \beta_{1-m i d}+b_{4} \cot \beta_{2-m i d} \\
& +b_{5} \cot \beta_{1-s h r}+b_{6} \cot \beta_{2-s h r}+b_{7} \Delta \theta_{h u b}+b_{8} \Delta \theta_{\text {mid }}+b_{9} \Delta \theta_{s h r}+b_{10} \\
& \eta=c_{1} \beta_{1-h u b}+c_{2} \beta_{2-h u b}+c_{3} \beta_{1-m i d}+c_{4} \beta_{2-m i d}+c_{5} \beta_{1-s h r}+c_{6} \beta_{2-s h r} \\
& +c_{7} \Delta \theta_{h u b}+c_{8} \Delta \theta_{\text {mid }}+c_{9} \Delta \theta_{\text {shr }}+c_{10} \\
& P_{\min }=d_{1} \beta_{1-h u b}+d_{2} \beta_{2-h u b}+d_{3} \beta_{1-m i d}+d_{4} \beta_{2-m i d}+d_{5} \beta_{1-s h r}+d_{6} \beta_{2-\text { shr }} \\
& +d_{7} \Delta \theta_{h u b}+d_{8} \Delta \theta_{\text {mid }}+d_{9} \Delta \theta_{\text {shr }}+d_{10}
\end{aligned}
$$

where $a, b, c$ and $d$ are the coefficient which of values are listed as the following Table 5.

Although we know that they do not conform to such a simple linear relationship, we can summarize a general trend. When the coefficient in front of the parameter is positive, the performance parameter will increase with increasing of the blade shape parameter. When the absolute value of the coefficient is large, it indicates that this parameter has a large weight.

\subsection{The Relationship Among Impeller Performance Parameters}

Generally, the most important index to evaluate the quality of a centrifugal pump is efficiency. In this section, the relationship diagram among performance parameters is drawn, as shown in Fig. 9. These points represent the relative relationship among performance parameters, where black represents the prediction results of ANN and red represents the calculation results of CFD. A note is that there is a proportional relationship among them. The relevance is that reaction force $F_{z}$ is small when efficiency $\eta$ is high, as shown in Fig.9(a). Head $H$ is small when efficiency $\eta$ is high, as shown in Fig. 9(b). The relationship is not obvious between efficiency $\eta$ and the minimum pressure $p_{\text {min }}$.

Table 5. The coefficients of polynomial formulation for impeller performance

\begin{tabular}{cc|cc|cc|cc}
\hline Coefficient & Value & Coefficient & Value & Coefficient & Value & Coefficient & Value \\
\hline$a_{1}$ & -0.072 & $b_{1}$ & 0.781 & $c_{1}$ & $-3.46 \mathrm{E}-04$ & $d_{1}$ & 0.426 \\
$a_{2}$ & -0.006 & $b_{2}$ & -1.299 & $c_{2}$ & $3.29 \mathrm{E}-04$ & $d_{2}$ & 0.464 \\
$a_{3}$ & -0.029 & $b_{3}$ & 0.528 & $c_{3}$ & $-3.94 \mathrm{E}-04$ & $d_{3}$ & -12.768 \\
$a_{4}$ & 0.218 & $b_{4}$ & -3.514 & $c_{4}$ & $-3.84 \mathrm{E}-04$ & $d_{4}$ & 5.465 \\
$a_{5}$ & -0.015 & $b_{5}$ & 0.342 & $c_{5}$ & $6.39 \mathrm{E}-05$ & $d_{5}$ & -7.567 \\
$a_{6}$ & -0.019 & $b_{6}$ & -0.612 & $c_{6}$ & $1.77 \mathrm{E}-05$ & $d_{6}$ & 1.316 \\
$a_{7}$ & -0.121 & $b_{7}$ & 0.061 & $c_{7}$ & $5.19 \mathrm{E}-04$ & $d_{7}$ & -0.771 \\
$a_{8}$ & 0.041 & $b_{8}$ & -0.110 & $c_{8}$ & $-6.85 \mathrm{E}-04$ & $d_{8}$ & -0.464 \\
$a_{9}$ & 0.125 & $b_{9}$ & -0.063 & $c_{9}$ & $-2.10 \mathrm{E}-04$ & $d_{9}$ & 6.777 \\
$a_{10}$ & 13.387 & $b_{10}$ & 121.445 & $c_{10}$ & 0.979 & $d_{10}$ & -786.438 \\
\hline
\end{tabular}

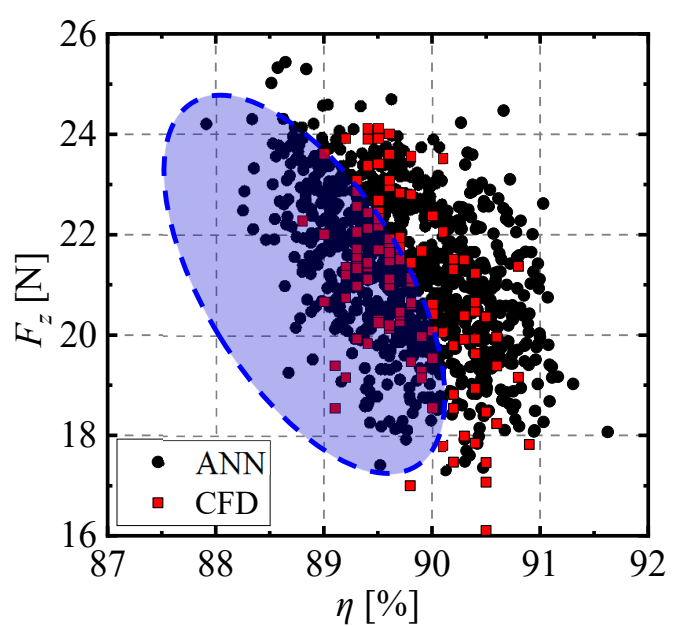

(a) Efficiency and reaction force

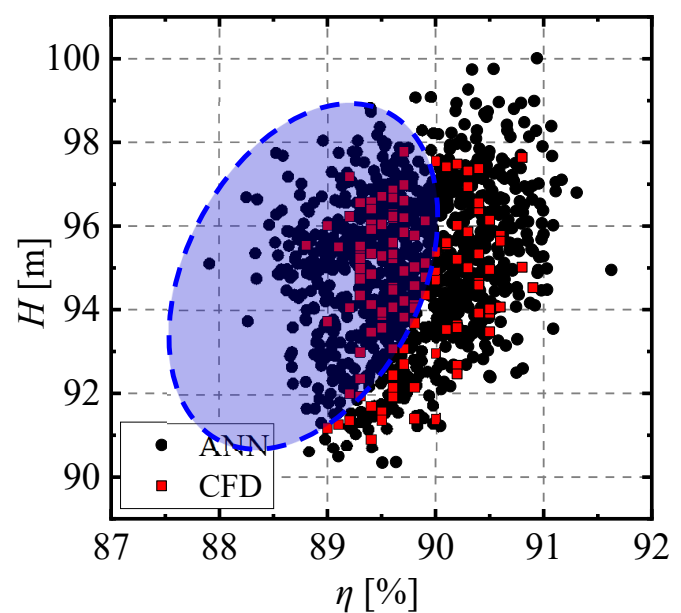

(b) Efficiency and head 


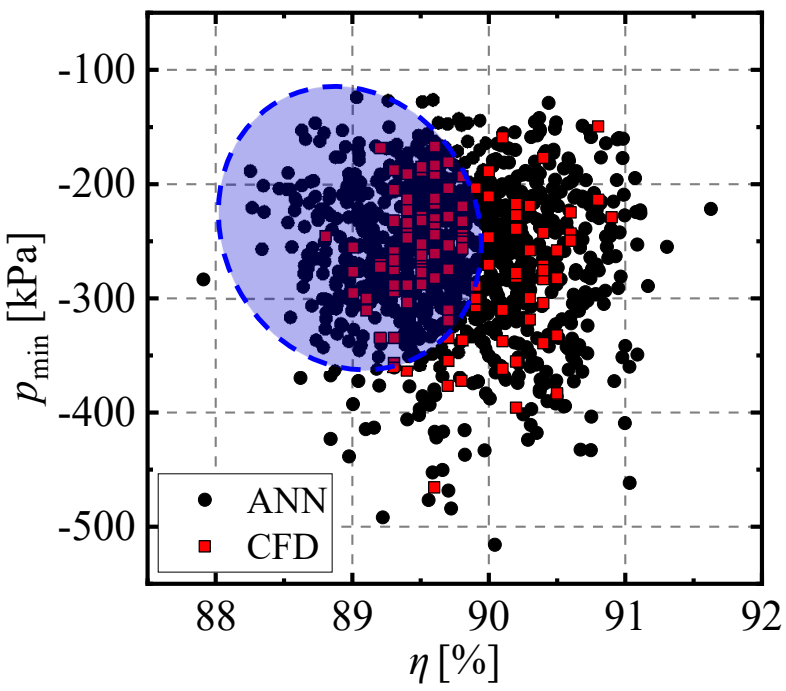

(c) Efficiency and the minimum pressure

Fig. 9. The Relationship Among the Impeller Performance Parameters

\section{Conclusion}

The influence of different blade angle and wrap angle on the performance of centrifugal pump was investigated by means of CFD and optimized ANN with a proof experiment. The influencing degree of blade shape parameters on the impeller performance were analyzed by GRA in detail. The conclusions are drawn as follows:

(a) The optimized ANN strategy was proposed. Based on 100 blade samples, many samples were calculated through this network. Compared with the CFD calculation results, it was found that the samples calculated by ANN also have high accuracy for the performance parameter of head, efficiency and reaction force. The optimized ANN is strongly effective. It provided a quick solution for many samples.

(b) Through gray correlation analysis, the sorts of parameters affecting performance parameters were found out. The blade angles at the leading edge were more influential for reaction force, head and minimum pressure. While the wrap angles have greater impact for efficiency. For further analysis, a multiple linear regression model was established. The absolute value of the coefficient represents the weight of the parameter.

(c) The relationship diagrams between efficiency and the other performance parameters were plotted with results by CFD and ANN. It can be summed up that efficiency is in roughly negative proportion to reaction force, while it is positive proportional to the head value. A certain performance parameter of a centrifugal pump has remarkable effects on the other performance parameter in a centrifugal pump, and more attention should be paid to them.

\section{Acknowledgement}

The authors would like to acknowledge the financial support of the National Natural Science Foundation of China. This study is funded by National Natural Science Foundation of China grant number 52079142; National Natural Science Foundation of China grant number 51836010.

\section{References}

Badr, H. M., \& Ahmed, W. H. (2015). Pumping machinery theory and practice. John Wiley \& Sons.

Celik, I. B., Ghia, U., Roache, P. J., \& Freitas, C. J. (2008). Procedure for estimation and reporting of uncertainty due to discretization in CFD applications. Journal of fluids Engineering-Transactions of the ASME, 130(7).

De Souza, B., Niven, A., \& Daly, J. (2008). Single-blade impeller development using the design of experiments method in combination with numerical simulation. Proceedings of the Institution of Mechanical Engineers, Part E: Journal of Process Mechanical Engineering, 222(3), 135-142.

Guan, X. F. (2011). Modern pumps theory and design. China Astronaut. Beijing China, 35-37.

Hou, F., Zhao, Z., Yu, Z., \& Xing, Z. (2017). Experimental study of the axial force on the rotors in a twin-screw refrigeration compressor. International Journal of Refrigeration, 75, 155-163.

Ju-long, D. (1982a). Grey control system. Journal of Huazhong University of Science and Technology, 3(9), 18.

Ju-Long, D. (1982b). Control problems of grey systems. Systems \& control letters, 1(5), 288-294. 
Larsen, B. E., \& Fuhrman, D. R. (2018). On the over-production of turbulence beneath surface waves in Reynolds-averaged Navier-Stokes models. Journal of Fluid Mechanics, 853, 419-460.

Launder, B. E., \& Sharma, B. I. (1974). Application of the energy-dissipation model of turbulence to the calculation of flow near a spinning disc. Letters in heat and mass transfer, 1(2), 131-137.

Menter, F. (1993, July). Zonal two equation kw turbulence models for aerodynamic flows. In 23rd fluid dynamics, plasmadynamics, and lasers conference (p. 2906).

Menter, F. R., Kuntz, M., \& Langtry, R. (2003). Ten years of industrial experience with the SST turbulence model. Turbulence, heat and mass transfer, 4(1), 625-632.

Nishi, Y., Fukutomi, J., \& Fujiwara, R. (2012, November). Effect of blade outlet angle on radial thrust of single-blade centrifugal pump. In IOP conference series: earth and environmental science (Vol. 15, No. 7, p. 072039). IOP Publishing.

Olsson, A., Sandberg, G., \& Dahlblom, O. (2003). On Latin hypercube sampling for structural reliability analysis. Structural safety, 25(1), 47-68.

Sen-Chun, M., Zhi-Xiao, S., Xiao-Hui, W., Feng-Xia, S., \& Guang-Tai, S. (2020). Impeller meridional plane optimization of pump as turbine. Science progress, 103(1), 0036850419876542.

Shi, W., Li, Q., Lu, W., \& Zhang, D. (2009). Estimation and experiment of axial thrust in centrifugal pump based on CFD. Nongye Jixie Xuebao= Transactions of the Chinese Society for Agricultural Machinery, 40(1), 60-63.

Wang, J. M., Wang, P. F., Zhang, X., Ruan, X. D., \& Fu, X. (2019). An adjoint-based optimization method for reducing the axial force of a reactor coolant pump. Journal of Zhejiang University-SCIENCE A, 20(11), 852-863.

Wilcox, D. C. (1988). Reassessment of the scale-determining equation for advanced turbulence models. AIAA journal, 26(11), 1299-1310.

Zhu, D., Xiao, R., Yao, Z., Yang, W., \& Liu, W. (2020). Optimization design for reducing the axial force of a vaned mixedflow pump. Engineering Applications of Computational Fluid Mechanics, 14(1), 882-896.

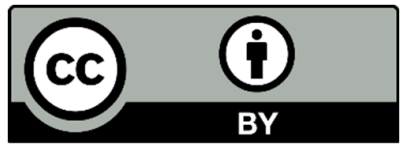

(C) 2022 by the authors; licensee Growing Science, Canada. This is an open access article distributed under the terms and conditions of the Creative Commons Attribution (CC-BY) license (http://creativecommons.org/licenses/by/4.0/). 